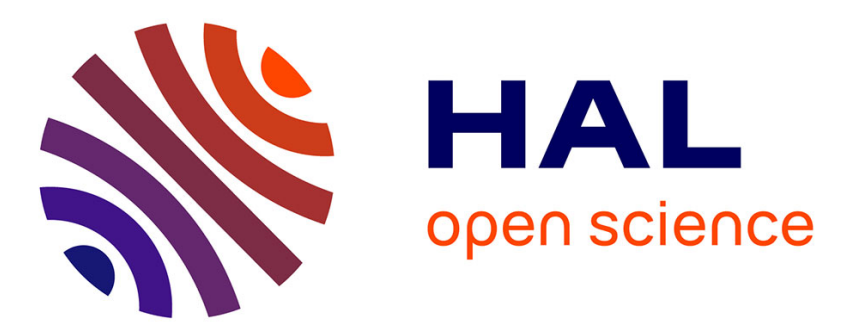

\title{
Biophysical properties of cardiomyocyte surface explored by multiparametric AFM
}

Georges Smolyakov, Marie Cauquil, Childérick Séverac, Véronique Lachaize, Céline Guilbeau-Frugier, Jean-Michel Senard, Céline Galés, Etienne Dague

\section{- To cite this version:}

Georges Smolyakov, Marie Cauquil, Childérick Séverac, Véronique Lachaize, Céline Guilbeau-Frugier, et al.. Biophysical properties of cardiomyocyte surface explored by multiparametric AFM. Journal of Structural Biology, 2017, 198 (1), pp.28-37. 10.1016/j.jsb.2017.03.001 . hal-01907685

\section{HAL Id: hal-01907685 https://hal.laas.fr/hal-01907685}

Submitted on 22 Nov 2018

HAL is a multi-disciplinary open access archive for the deposit and dissemination of scientific research documents, whether they are published or not. The documents may come from teaching and research institutions in France or abroad, or from public or private research centers.
L'archive ouverte pluridisciplinaire HAL, est destinée au dépôt et à la diffusion de documents scientifiques de niveau recherche, publiés ou non, émanant des établissements d'enseignement et de recherche français ou étrangers, des laboratoires publics ou privés. 


\section{Biophysical properties of cardiomyocyte surface explored by multiparametric AFM}

Georges Smolyakov ${ }^{\mathrm{a}, \mathrm{b}, \mathrm{e}}$, Marie Cauquil ${ }^{\mathrm{c}, \mathrm{e}}$, Childerick Severac $^{\mathrm{a}}$, Véronique Lachaize ${ }^{\mathrm{a}, \mathrm{b}, \mathrm{c}, \mathrm{e}}$, Céline Guilbeau-Frugier ${ }^{\mathrm{c}, \mathrm{d}, \mathrm{e}}$, Jean-Michel Sénard ${ }^{\mathrm{c}, \mathrm{e}}$, Céline Galés ${ }^{\mathrm{a}, \mathrm{c}, \mathrm{e}^{*}}$, Etienne Dague ${ }^{\mathrm{a}, \mathrm{b}, \mathrm{e}^{*}}$.



bLAAS-CNRS, Université de Toulouse, CNRS, France

'Institut des Maladies Métaboliques et Cardiovasculaires, INSERM UMR1048, Université de Toulouse, France

${ }^{\mathrm{d}}$ Centre de Microscopie Électronique Appliquée à la Biologie, Université de Toulouse, France

${ }^{\text {e }}$ Service de Pharmacologie, CHU de Toulouse, Université de Toulouse, Toulouse, France

*These authors contributed equally to this work and are corresponding authors.

Corresponding author 1: Dr Etienne Dague

Address: $\quad$ LAAS-CNRS, Université de Toulouse, CNRS, France,

7 av du Colonel Roche

31000 TOULOUSE, FRANCE

Phone: +33 561337841

Email: edague@laas.fr

Corresponding author 2: Dr Céline Galés

Address: INSERM UMR1048

Institut des Maladies Métaboliques et Cardiovasculaires - I2MC

1, avenue Jean-Poulhès - BP84225

31432 Toulouse cedex 4

France

Phone: +33 561324081

Email: celine.gales@inserm.fr 


\begin{abstract}
PeakForce Quantitative Nanomechanical Mapping (PeakForce QNM) multiparametric AFM mode was adapted to qualitative and quantitative study of the lateral membrane of cardiomyocytes (CMs), extending this powerful mode to the study of soft cells. On living CM, PeakForce QNM depicted the crests and hollows periodic alternation of cell surface architecture previously described using AFM Force Volume (FV) mode. PeakForce QNM analysis provided better resolution in terms of pixel number compared to FV mode and reduced acquisition time, thus limiting the consequences of spontaneous living adult CM dedifferentiation once isolated from the cardiac tissue. PeakForce QNM mode on fixed CMs clearly visualized subsarcolemmal mitochondria (SSM) and their loss following formamide treatment, concomitant with the interfibrillar mitochondria climbing up and forming heaps at the cell surface. Interestingly, formamide-promoted SSM loss allowed visualization of the sarcomeric apparatus ultrastructure below the plasma membrane. High PeakForce QNM resolution led to better contrasted mechanical maps than FV mode and provided correlation between adhesion, dissipation, mechanical and topographical maps. Modified hydrophobic AFM tip enhanced contrast on adhesion and dissipation maps and suggested that CM surface crests and hollows exhibit distinct chemical properties. Finally, two-dimensional Fast Fourier Transform to objectively quantify AFM maps allowed characterization of periodicity of both sarcomeric Z-line and M-band. Overall, this study validated PeakForce QNM as a valuable and innovative mode for the exploration of living and fixed CMs. In the future, it could be applied to depict cell membrane architectural, mechanical and chemical defects as well as sarcomeric abnormalities associated with cardiac diseases.
\end{abstract}




\section{Introduction}

Atomic force microscopy (AFM) (Binnig et al., 1982; Binnig and Quate, 1986) is a powerful tool for nanoscale research in biology (Muller et al., 2009; Dufrêne et al., 2008; Cross et al., 2007; Liu and Wang, 2010; Pillet et al., 2014). Since the development of the Force Volume (FV) AFM mode in 1994 (Radmacher et al., 1994), it became possible to obtain the force curves related to the interaction between the AFM tip and the sample surface with a spatial resolution. In this particular mode, the tip approaches the surface, comes in contact and presses until a predefined force, called peak force, is reached. Then, the tip is retracted and moves to the next position. The force curves thus measured allow extracting the topography, as well as the mechanical and adhesive properties of the sample (Fig. 1A). Hence, classical AFM analysis gives access to both structural and mechanical information from the same force curves (Formosa et al., 2012a; Formosa et al., 2012b).

Recently, pulsed force (Krotil et al., 1999) and multiparametric AFM modes were developed (Hu et al., 2011; Alsteens et al., 2012; Dufrêne et al., 2013; Martín-Rodríguez et al., 2014; Formosa et al., 2015; Chopinet et al., 2013 ; Smolyakov et al., 2016). In these modes, the tip pathway is modified, allowing faster acquisition of force curves (Fig. 1B) and sample imaging with high resolution $\left(\geq 128^{2}\right.$ pixels) in reasonable time scale (5-10 min) (Fig. 1C). This is not the case in the FV mode, due to a negative relationship between resolution level and acquisition time because of the low tip oscillation frequency. In the PeakForce QNM multiparametric mode developed by Bruker Corporation, the scanning parameters can be adjusted according to that each pixel value and data represent an average over several force curves whereas in the FV, each pixel value relates to only one force curve. Nevertheless, today, experience of PeakForce QNM for analysis of soft mammalian cells remains rather limited relying essentially on two reasons. First, the oscillation amplitude of the cantilever in this mode (currently up to $4 \mu \mathrm{m}$ ) remains lower compared to the FV $(7.5 \mu \mathrm{m})$ thus potentially leading to incomplete tip retraction on very soft and adhesive cells. Second, the very high frequency of tip oscillations ( $\geq 250 \mathrm{~Hz})$ may result in viscoelastic effects (Costa, 2003; Guilak, 2000; Huang et al., 2004) and in mechanical behaviour not consistent with that observed with FV mode in quasi-static conditions (Smolyakov et al., 2016). It follows that the adaptation of PeakForce QNM for mammalian cells analysis still remains challenging (Slade et al., 2014; Hozic et al., 2012; Heu et al., 2012; Calzado-Martín et al., in press).

Adult cardiomyocytes (CMs), the contractile cellular entity of the heart, are highly differentiated mammalian cells measuring around 100-150 $\mu \mathrm{m}$ in length and 10-20 $\mu \mathrm{m}$ in width and exhibiting a typical rod-shape (Leu et al., 2011). Once isolated from the cardiac tissue, these non proliferative cells are highly unstable and quite fragile, thus requiring specific culture medium conditions and accurate temperature and $\mathrm{CO}_{2}$ controlled atmosphere to stabilize their structure and viability over time. However, even in suitable culture conditions, CMs tend to spontaneously and quite rapidly 
dedifferentiate, thus loosing progressively their rod-shape (time scale range from minutes to several hours) (Dague et al., 2014). Moreover, CMs are relatively soft (elastic modulus $<20 \mathrm{kPa}$ ) and highly adhesive to the AFM tip, susceptible to mechanical stress, and often contracting under mechanical or light stress even in the presence of contraction inhibitors. These characteristics make CMs challenging cells for AFM study requiring optimization of both the resolution and the acquisition time of AFM experiments. It is therefore not surprising that high resolution analysis of the living CMs surface using AFM is quite rare. Only few studies were performed on living CMs but they were not performed under controlled atmosphere and did not take into account cell dedifferentiation time following isolation (Lieber 2004, Benech 2014, Benech 2015). Despite these studies provided information about the global changes of CM elasticity, they did not inform about the topographic organization of the cell surface neither its accurate elasticity correlation.

The present study follows a previous work from our group (Dague et al., 2014) in which we used AFM FV mode to explore the topography and elasticity of the cell surface (lateral membrane) of living adult CMs and their potential modifications in heart failure following myocardial infarction in mice. This study revealed an extremely organized structure of CMs cell surface with periodic alternation of stiff crests linked to the presence of subsarcolemmal mitochondria (SSM) and soft hollows. Interestingly, shortly after infarction, CMs loose SSM resulting in cell surface smoothing and decreased elastic modulus. At long-term stage, interfibrillar mitochondria (IFM) migrate towards the surface, creating heaps and underlying general increase of the elastic modulus.

In the present work, we adapted for the first time the PeakForce QNM mode to both living and fixed adult CMs to study the topography and the mechanical properties of their lateral membrane. The living CMs are among the softest cells ever explored quantitatively by the PeakForce QNM and their fixation by chemical agents increases their relative stiffness but also stabilizes CMs morphology immediately after purification thus preventing cells from dedifferentiation, and allowing AFM measurements under non restrictive atmosphere/temperature conditions. The use of PeakForce QNM led to a significant decrease of the acquisition time, as well as to increased resolution in terms of pixels number and in terms of statistical significance of every pixel of the CMs surface maps. Moreover, both the adhesion and dissipation maps of the CM cell surface with well-defined contrast, not accessible in the FV mode due to its low resolution and tip oscillation frequency, could be further delineated by PeakForce QNM. The adhesion map represents the chemical surface contrast, while the dissipation map contrast may be considered as combination of chemical and viscous contributions. The use of modified hydrophobic tips (Dague et al., 2007; Dague et al., 2008; Alsteens et al., 2007) increased the contrast for the adhesion and dissipation maps, allowing us to depict very accurate structures of the CM surface, as well as to determine its relative chemical character. We also proposed in this study to use a two-dimensional Fast Fourier Transform (FFT) (Cooley and Tukey, 1965) to analyze the periodicity of the surface structures of the CM from the different maps. Finally, we used 
PeakForce QNM mode on fixed CMs to study alterations of the cell surface structure induced by formamide treatment that we previously demonstrated to phenocopy those observed following infarction (Dague et al, 2014). Taken as a whole, our study indicates that the PeakForce QNM provides many advantages for the study of CMs structural, mechanical and adhesive properties. 


\section{Materials and methods}

\subsection{CMs isolation (Figure 2)}

Adult CMs were isolated from 2-month old C57/B16 mice as previously described using the Langendorff method (Dague et al., 2014) (Fig. 2A). After isolation, living CMs were plated on laminin-coated glass Petri dishes at $37^{\circ} \mathrm{C}$ in $5 \% \mathrm{CO}_{2}$ atmosphere in resuspension solution containing $10 \%$ bovine calf serum, $12.5 \mu \mathrm{M} \mathrm{CaCl}_{2}$ and $10 \mathrm{mM}$ BDM (2,3-butanedione monoxime) in order to inhibit spontaneous cell beating.

For the study of living CMs, the resuspension medium was then changed to MEM culture medium containing $1 \%$ penicillin-streptomycin-glutamine, $4 \mathrm{mM} \mathrm{NaHCO}_{3}, 10 \mathrm{mM}$ Hepes, $0.2 \%$ BSA and 25 $\mu \mathrm{M}$ blebbistatin. The Petri dish was transferred to the AFM microscope and cells were subjected to the $\mathrm{AFM}$ experiment in a controlled chamber at $37^{\circ} \mathrm{C}$ in $5 \% \mathrm{CO}_{2}$ atmosphere.

For the study of fixed CMs, after plating, cells were washed twice in the resuspension medium and fixed in ice-cold methanol for 10 minutes. Fixed CMs were further subjected to AFM experiment at ambient atmosphere.

\subsection{Formamide treatment of $C M s$}

CMs were submitted to formamide-induced osmotic shock in order to induce mitochondrial shift as previously described (Dague et al, 2014). Briefly, after isolation and plating, CMs were incubated in resuspension solution (See 2.1) containing $0.25 \mathrm{M}$ (low concentration) or $0.75 \mathrm{M}$ (high concentration) formamide for 10 minutes at $37^{\circ} \mathrm{C}$ in $5 \% \mathrm{CO}_{2}$ atmosphere. The cells were then washed with resuspension solution and fixed with ice-cold methanol for 10 minutes. Fixed CMs were washed with PBS and maintained in PBS until AFM analysis.

\subsection{Conditions of AFM experiments}

The AFM study was performed on the Bioscope Catalyst (Bruker Corporation). The choice of scanning parameters was the major step to adapt the PeakForce QNM for the soft and adhesive living CMs. In order to ensure complete tip withdrawal and to minimize viscoelastic effects, the maximum oscillation amplitude $(4 \mu \mathrm{m})$ and the minimum frequency $(250 \mathrm{~Hz})$ were respectively applied. The applied peak force $(1 \mathrm{nN})$ was chosen to detect a mechanical contrast between different structural elements but avoiding the high adhesion and incomplete tip retraction. The images resolution was $128^{2}$ pixels, the scan size was $10^{2} \mu \mathrm{m}^{2}$. As for the line rate, it can vary between $0.2-0.5 \mathrm{~Hz}$, depending on the cell state, which influences the adhesion between tip and surface. The comparative FV 
measurements were conducted using the same amplitude, peak force and scan size as for the PeakForce QNM analysis, but the tip frequency was $6 \mathrm{~Hz}$ and resolution was $64^{2}$ pixels. The same PeakForce QNM parameters adapted for the living CMs were used to analyze fixed CMS. In these fixed cells, the line rate was kept constant $(0.5 \mathrm{~Hz})$ as the scanning is more stable compared to the living CMs.

\subsection{AFM cantilevers}

Two different cantilevers were used for living CMs. First, the MLCT-C cantilever with the nominal spring constant of $10 \mathrm{mN} / \mathrm{m}$. The spring constant was systematically measured by means of the thermal tune (Emerson and Camesano, 2006). The nominal radius of the corresponding non modified tip was $20 \mathrm{~nm}$. Second, for the hydrophobic interaction measurements (Dague et al., 2007; Dague et al., 2008; Alsteens et al., 2007) the NPG-10-D cantilever was applied, with the nominal spring constant of $60 \mathrm{mN} / \mathrm{m}$ and the nominal tip radius of $30 \mathrm{~nm}$. In order to provide the hydrophobic character for this gold-coated NPG-10-D cantilever, the latter was immersed for $14 \mathrm{~h}$ in $1 \mathrm{mM}$ $\mathrm{HS}\left(\mathrm{CH}_{2}\right)_{11} \mathrm{CH}_{3}$ (Sigma-Aldrich) in ethanol, then rinsed with ethanol and dried with nitrogen. As fixed CMs are stiffer compared to the living ones, only the NPG-10-D cantilevers were used in this case.

\subsection{Analysis of AFM data}

As noted above, in the case of living CMs, the deformation was much larger compared to the tips radius. Thus, the Sneddon model for conical contact was applied in order to estimate the elastic modulus (Sneddon, 1965). The Sneddon model links the sample deformation $d$ to the applied force $F$ via the elastic modulus $E$ and the tip half-angle $\alpha$ values: $F=2 / \pi^{*} E /\left(1-v^{2}\right)^{*} \tan \alpha^{*} d^{2}$, with $\alpha$ equal to $17.5^{\circ}$ and $v$ being the sample Poisson ration, arbitrary taken as 0.5 .

For the fixed CMs, the deformation was around $100 \mathrm{~nm}$ and the nominal diameter of NPG-10-D tips is equal to $60 \mathrm{~nm}$, being further slightly increased due to the thiol layer grafted to the tip surface, making the Sneddon model no longer valid. Thus, the Derjaguin-Muller-Toporov (DMT) model for the spherical tip-surface contact was applied (Derjaguin et al., 1975; Muller et al., 1983). This model links $d$ to $F$ via $E$ and tip radius $R$ values, by taking into account adhesion force $F_{a d h}$ as a contribution to the effective force: $F=4 / 3 * E /\left(1-v^{2}\right) * R^{1 / 2} * d^{3 / 2}+F_{a d h}$. To account for the adhesion, the retract force curve is fitted by this model.

The force curves in PeakForce QNM experiment were analyzed online, while in the FV measurements the curves were collected and then treated by SPIP software with the correction stage consisted in the noise reduction and the base line adjust. 
In order to decompose the harmonic components of AFM signal, the Gwyddion software was used for image analysis by means of two-dimensional Fast Fourier Transform (2D-FFT) applied to series of data of arbitrary dimensions without resampling. Data are expressed as spectral modulus corresponding to the square root of the power spectrum density. Filtering was applied to exclude potential linear asymmetry and Hann windowing function was used to suppress the data at the edges of the image.

\subsection{Transmission Electron Microscopy}

Heart tissue samples were fixed with $2 \%$ glutaraldehyde in Sorensen's buffer and proceeded as described in Genet et al (Genet et al., Circ Res 2012).

\subsection{Statistical analysis}

Results are depicted as mean \pm S.E.M. of 8-12 CMs from 3 mice performed on 3 different experiments. Comparisons were achieved using one-way ANOVA when appropriate. A $P$ value $<0.05$ was considered as significant. 


\section{Results and Discussion}

\subsection{PeakForce QNM versus FV analysis of living CMs}

As discussed in subchapter 2.5., two different cantilevers (MLCT-C and NPG-10-D) were applied in this study. The use of modified NPG-10-D cantilever, due to the different surface character of the tip, only changed adhesion and dissipation compared to the MLCT-C cantilever. Both cantilevers possess the spring constant in an appropriate range (the cantilever deflection and the sample deformation are of the same order of magnitude). In its turn the tip radius in both cases is negligible with respect to the deformation ( 700 nm). Thus, both MLCT-C and modified NPG-10-D tips gave approximately the same results (with a difference below the AFM measurements error) for the topography and elastic modulus. The latter was calculated from the approach curve, thus minimizing the adhesion effect.

Representative results of the PeakForce QNM on a living CM are shown in Fig. 3. The hydrophobic tip was used for these experiments in order to increase the contrast for the adhesion and dissipation. The acquisition time for a PeakForce QNM measurement was about 5-7 min for $10^{2} \mu \mathrm{m}^{2}$ scan size with $128^{2}$ pixels resolution, while it was equal to $10 \mathrm{~min}$ in the FV for the same scan size, but with lower, $64^{2}$ pixels resolution. As shown in Fig. 3B, the PeakForce QNM force curves preserve indentation region for height measurements, mechanical or adhesion characteristics. At the same time, the non contact part of the curves is far away from flat. This could potentially be related to the viscosity of the aqueous medium inducing at very high speed of piezo displacement virtual repulsive forces while approaching and virtual attracting forces while retracting. Thus, the viscous medium may lead to the cantilever deflection during its fast oscillation. This hypothesis could explain as well the fact that the approach step takes longer times compared to the retraction one, and this is related only to the non contact part of the curves. When the tip and surface are in contact, the approach and withdraw parts of the curves become symmetrical with respect to the time. Indeed, according to this hypothesis, once reaching the contact point (the tip is fixed on a surface), this medium effect should decrease, if not disappear.

As shown, the PeakForce QNM provided a high structural contrast in terms of cell surface topography (Fig. 3C), as well as of elasticity (Sneddon elastic modulus, Fig. 3D), adhesion (Fig. 3E) and dissipation (Fig. 3F) when compared to the FV mode conducted in similar conditions on the same CM (Fig. 4). For the topography, despite the high resolution of PeakForce QNM, the sharpness of the image is not perfect, and a number of artefacts are present. Indeed, as the tip oscillates very quickly in this mode, a precise contact point determination on such soft CM's samples is rather complicated. On the contrary, the elasticity map displays very good quality without pixel artefact (Fig. 3D) and a better contrast between different structural elements of the surface compared to the FV mode used in this 
study (Fig. 4D). The average Sneddon elastic modulus for the whole $10^{2} \mu \mathrm{m}^{2}$ scanned area was $5.8 \pm$ $2.8 \mathrm{kPa}$ ( 5 cells; 3 mice). This is quite different from the study by Dague et al (Dague et al., 2014), in which the authors stated an average modulus for the living $\mathrm{CM}$ of $16.7 \pm 7.1 \mathrm{kPa}$ following similar CM preparation. These discrepancies between the two studies most probably arise from different scanning conditions (applied force and tip oscillation frequency), elastic model as well as the cell state that can highly fluctuate due to natural dedifferentiation of the living adult CMs as stated above.

Interestingly, adhesion force and the dissipation energy maps could be obtained with well-defined contrast by PeakForce QNM (Fig. 3E, F). These images were subjected to the "Flatten" correction for better visualisation of the contrast. As shown in Fig. 3G, a good correlation could be observed between adhesion, dissipation, elasticity and topography maps.

FV analysis was carried out on living CMs (Fig. 4) using the same oscillation amplitude and peak force as well as the same hydrophobic tip like for the PeakForce QNM scanning. The same cell surface area of the CM was probed using the two modes (Fig. 3 and Fig. 4). The results indicate that FV analysis resulted in topography maps with well-defined periodic structures (Fig. 4C). The Sneddon elastic modulus map displayed small contrast but a clear correlation with the height map (Fig. 4D). However, compared to the PeakForce QNM, the adhesion and dissipation demonstrated no contrast between structural elements (Fig. 4E, F) most probably due to the very poor statistical significance of each pixel (based on one force curve) in the FV, as well as low tip oscillation frequency.

\subsection{PeakForce QNM analysis of fixed CMs: examination of formamide-induced cell surface} architectural defects.

Once validated PeakForce QNM in adult living CMs at resting state, this mode was applied for its potential to explore their architectural cell surface defects that can be found in pathological conditions. Specifically, we decided to use chemical treatment with formamide that was previously validated to reproduce the loss of cell surface crests and hollows and the mitochondrial shift observed in CMs following myocardial infarction (Dague et al 2014). To prevent any cell surface mitochondrial defects due to the living cell natural dedifferentiation or stringency of heart digestion, fixed CMs were used for the AFM investigation. Depending on the formamide concentration, differential impact on the CM structural surface can be achieved as previously shown (Dague et al., 2014). Relatively low formamide concentration $(0.25 \mathrm{M})$ promoted the SSM death, leading to the CM surface smoothing. At higher concentration $(0.75 \mathrm{M})$, formamide led to further alteration of the interior of the cell with the IFM migration towards the surface resulting in heaps formation. Fig. 5 displays comparative PeakForce QNM study of fixed control CMs exposed or not to different formamide concentrations. In non formamide-treated fixed CMs (control) (Fig. 5A), regular crests periodicity structured by SSM (Fig. 
5Aa), as well as a nice correlation between structure and mechanical properties were observed (Fig. 5Ae). Crests are always stiffer and less adhesive compared to the hollows. Some of the cells exhibit crests height up to $800 \mathrm{~nm}$, which is in agreement with the transmission electron microscopy images (Dague et al., 2014). Excellent resolution of PeakForce QNM allows visualizing directly (Fig. 5A) that the crests in the fixed control CMs are formed by under-membrane rounded structures in agreement with the presence of mitochondria. Imprinting of these rounded mitochondria can also be very well deduced from adhesion and dissipation maps (Fig. 5Ac, d), Indeed, the hollows are more adhesive with respect to the crests, indicating different chemical properties of the cell membrane between crests and hollows. On one hand, as expected, the adhesion and the dissipation are correlated, since adhesion largely contributes to dissipation. On the other hand, the contrast is better for the dissipation compared to the adhesion. The last observation could be explained by the contribution of the viscoelastic response to dissipation, as well as adhesion does. The energy loss due to the viscous behaviour should be higher for the softer zones (hollows) compared to the harder ones (crests), which are more elastic (being harder in the same time). Thus, the differences in terms of adhesion, as well as in terms of viscoelastic behaviour, going in the same direction, provide high contrast for the dissipation. As the hollows on CMs surface are more adhesive with respect to the hydrophobic tip compared to the crests, it indicates that hollows are more hydrophobic.

It is quite puzzling that overall PeakForce QNM analysis of adult living CMs (Fig. 3) differs from similar analysis but on fixed CMs (Fig. 5A). The most evident difference is the presence of mitochondria imprinting visualized on the dissipation map obtained from fixed cells (Fig. 5Ad) that is completely absent from the same analysis on living cells. Moreover, clear differences in crest heights between fixed and living CMs could also be noticed with higher measurements in fixed cells, consistent with the presence of mitochondria as described in (Dague et al., 2014). Overall, these results most likely argue for the dedifferentiation process of the living CMs that lead to the natural loss of crest and associated SSM.

In Fig. 5Ba (topography), cell surface smoothing due to the loss of SSM was observed, thus confirming the action of low formamide concentration treatment. More interestingly, it was possible in all AFM maps (Fig. 5B) to distinguish subsarcolemmal myofibrills as well as periodic sarcomeric organization along longitudinal myofibrils. It is interesting to notice that these maps are highly similar to those obtained with living CMs (Fig. 3), thus confirming the dedifferentiation process with loss of cell surface crests and SSM. Following high formamide concentration treatment (Fig. 5Ca), heaps could be observed confirming the migration and the superimposition of IFM beneath cell surface that we previously described (Dague et al, 2014). Despite relatively low oscillation amplitude in PeakForce QNM (compared to the FV mode), large heaps with heights up to $1.4 \mu \mathrm{m}$ were easily scanned without any image artefacts. Moreover, the elastic modulus appeared one order of magnitude higher for the fixed cells compared to the living ones. In particular, we measured an average elastic modulus on the 
whole map of $108 \pm 9 \mathrm{kPa}$ for control cells, $69 \pm 6 \mathrm{kPa}$ for low formamide concentration treatment in agreement with the SSM death, and $118 \pm 16 \mathrm{kPa}$ for high formamide concentration treatment $(P<$ 0.05 control vs low concentration, $P<0.05$ low concentration vs high concentration) confirming the cell surface accumulation of IFM heaps. Although the absolute modulus values are largely increased for fixed cells, their relative variations upon formamide treatment are relatively preserved as described for the living CMs (Dague et al., 2014).

\subsection{Dissipation energy allows depicting ultrastructural components of the contractile myofibrills}

As noticed above, an advantage of the use of PeakForce QNM for the analysis of the CM cell surface was to provide enough contrast for the adhesion and dissipation maps, which are together correlated (with better contrast for the dissipation). Surprisingly, under exposure to low formamide concentration leading to the CM surface smoothing, these dissipation maps (as well as the adhesion ones) gave access to accurate details of the sarcomere structure on the bottom of the membrane that FV mode was unable to depict (Fig. 6A). Indeed, from traces on the dissipation maps (Fig. 6A), periodically spaced $(\sim 2 \mu \mathrm{m})$ "camel" double-crests structures were identified. This $\sim 2 \mu \mathrm{m}$ space is in agreement with the size of the sarcomere (contractile entity of the myofibrills) measured using transmission electron micrographs (Fig. 6B). On this image, the sarcomeric structure is delimited by two Z-lines $(\sim 1.5-2 \mu \mathrm{m})$ with the M-band localized in the middle $(\sim 1 \mu \mathrm{m}$ size $)$ which probably corresponds to the medium hollow on the traces from dissipation maps. This result indicates that the chemical properties of the Z-line and the M-band are quite distinct. Each myofibrill is composed of a succession of sarcomeres thus alternating successively Z-lines and M-bands as most probably observed on the dissipation traces. When the surface of the CM was scanned at smaller scale so to more accurately visualize structural details, the height map (Fig. $6 \mathrm{Cb}$ ) resulting from a $2.2^{2} \mu \mathrm{m}^{2}$ zoom allowed identifying the presence of the contractile myofibrils-forming myofilaments. Up to now, such CM ultra-structures could only be visualized using electron microscopy resolution. The presence of myofilaments is even more clearly observed on the PeakForce error map (Fig.6Cc) which corresponds to the deviation of the real peak force from the requested one. This deviation is very sensitive to the mechanical behaviour of the sample, and although it cannot be directly interpreted in terms of sample properties, it often provides excellent structural contrast. 


\subsection{Periodicity of the sarcomere structure analysed by Fast Fourier Transform}

Two-dimensional Fast Fourier Transform (2D-FFT) was used to analyse the periodicity of CM sarcomere on SSM-depleted CM obtained following low formamide concentration treatment on fixed CMs (Fig. 7). The FFT allows exploring the whole AFM images, providing frequency, periodicity as well as spectral modulus of CM membrane events on different PeakForce QNM maps. As shown on the Fig. 7, the AFM image (in the real space) (left panels) is transformed to the corresponding 2D-FFT image (the invert space) (middle panels). This latter displayed correlations between structural elements that were further traced in order to compute the corresponding characteristic lengths (Fig. 7, right panels). The graph of the corresponding trace is symmetrical with respect to a central peak and contains a number of correlation peaks to the left or to the right of the central reference peak.

Simultaneous analysis of QNM PeakForce maps and of corresponding 2D-FFT clearly indicates that, whatever the AFM map considered, spectral peaks are separated by a constant periodic distance (Fig. 7). From correlation with AFM maps, the $d$ distance corresponds to the spacing between neighbour peaks. Intermediate peaks are separated by a distance equal to $2 d(2.04 \pm 0.04 \mu \mathrm{m})$ in agreement with the sarcomere size (Fig. 6B) delimited by two Z-lines, while the location of the Mband in between of two Z-lines is responsible for the $d$ distance correlation $(1.02 \pm 0.02 \mu \mathrm{m})$. The intensity of the correlation peak may be considered as a measure of the image contrast, while the intensity of the alternation peak over the correlation peak (degree of alternation) ratio is related to the contrast between signals from Z-line and M-band. Concerning the height image (Fig. 7A), the correlation peak is rather small, as the quality of the height images is relatively low in the PeakForce QNM analysis. The alternation peak is present as well, indicating that a higher peak is followed by a smaller one. As for the DMT elastic modulus, the correlation between neighbour peaks is defined better compared to the height image. This demonstrates that PeakForce QNM provides better contrast for the elastic modulus compared to the topography map. The alternation peak is much less intense compared to the main correlation one. It means that Z-line and M-band regions exhibit approximately the same modulus despite different height. The alternation between Z-lines and M-bands was better defined on the adhesion map (Fig. 7C), while on the dissipation map this alternation profile is in between those for the elasticity and the adhesion maps. This was expected since both mechanical and adhesive behaviours contribute to the dissipation, as discussed above. Moreover, all 2D-FFT maps provided exactly the same $d$ distance between peaks (Z-line and M-band) (1.02 $\pm 0.02 \mu \mathrm{m})$. Thus, Zlines and M-bands are the most distinguishable in terms of adhesion, less in terms of height and display almost similar elastic behaviour. This discrimination between adhesion and elastic modulus is important to prove that observed differences in adhesion on CM surface are specific and not simply related to different surface deformation and consequently to contact area between the tip and cell 
surface. So, it appears that the adhesion contrast is not the simple reflection of the elastic contrast, but has its own specific origin.

In general, the problem of possible mutual influence of different explored properties is important. The specific profiles of corresponding FFT traces in Fig. 7 prove that height, elastic modulus and adhesion have their own physical origin, being not the artefacts of measurements. Special attention should be paid to the height variation influence on other properties. Indeed, the cardiomyocytes surface is not flat, which may lead to two potential artifacts. First, the tip-surface contact may deviate from symmetric form. But this effect has very minor importance in regard of huge deformation in living cardiomyocytes case and huge modulus variation across the surface for the fixed cells. Second, different height of structure elements could cause their different deformation. But this is not true for the PeakForce QNM, as it is a force-feedback mode and not an amplitude-feedback mode. So no the height of the structure does not matter, the tip stops indenting once the predefined force is reached.

In conclusion, it follows that the FFT provides a fast and powerful tool for quantitative analysis of the periodicity of the sarcomere Z-band and M-band on smooth SSM-depleted CMs from height, mechanical and adhesion/dissipation AFM-based images. 


\section{Conclusion}

The novel multiparametric PeakForce QNM AFM mode was adapted for the CMs study and further used to investigate changes in CM surface architecture induced by formamide and mimicking those observed after myocardial ischemia (Dague et al, 2014). Thus, we succeeded to extend the limits of this powerful mode to the quantitative study of softer cells.

On one hand, the use of PeakForce QNM for CMs provided higher resolution in terms of the pixels, as well as of statistical significance of every pixel with respect to the classical FV mode. On the other hand, the acquisition time in PeakForce QNM was dramatically decreased compared to the FV, which is extremely important for living CMs due to their rapid spontaneous dedifferentiation after isolation from the cardiac tissue.

PeakForce QNM was successfully used on fixed cells to follow the cell surface mitochondrial shift induced by a chemical agent. In these conditions, this AFM mode provided a highly resolutive tool to visualize ultrastructural (nanometer scale) components of the sarcomeric apparatus. Up to now, such structures could be only appreciated using transmission electron microscopy.

The use of PeakForce QNM resulted in higher contrast for the elastic modulus with respect to FV. More interestingly, the PeakForce QNM gave access to the adhesion and dissipation maps with welldefined contrast allowing observation of the most accurate details of the CM structure. In particular this analysis allowed us to clearly visualize SSM cell surface imprinting. These mitochondria were only described up to now from deduction of AFM height and elasticity data in combination with electron imaging. We showed that SSM/crests are less hydrophobic compared to the hollows. Moreover, dissipation maps in the absence of cell surface SSM depicted the periodic alternation of Zlines and M-bands composing the sarcomeric contractile apparatus. The adhesion maps identified different biochemical properties of the cell surface located at the Z-line or the M-band while the differences were smaller in terms of cell surface height and almost absent in terms of elastic modulus.

The FFT is proposed as a fast and accurate tool for the objective analysis of a whole AFM image, providing quantitative analysis of the periodicity between different structural components of the sarcomeric apparatus, i.e. the Z-line and the M-band. In the future, such analysis could be useful to accurately depict sarcomeric defects in cardiac diseases.

Finally, the PeakForce QNM proved that fixed CMs were better suited for preservation of the native and pathological cell surface structures.

Overall, our study shows that PeakForce QNM is an innovative tool for the AFM study of CMs that could be extended for the analysis of specific cell surface defects associated with heart diseases. 


\section{Acknowledgements}

This work was supported by the "Fondation pour la Recherche Médicale" (G.S.; grant ING21040129094) and Fondation Bettencourt Schueller (C.G.; Coup d'Elan pour la recherche Française 2013). ED is researcher at the Centre National de la Recherche Scientifique (CNRS) and CG is researcher at Institut National de la Santé et de la Recherche Médicale (INSERM) 


\section{References}

Alsteens, D., Dague, E., Rouxhet, P.G., Baulard, A.R., Dufrêne Y.F., 2007. Direct measurement of hydrophobic forces on cell surfaces using AFM. Langmuir 23, 11977-11979.

Alsteens, D., Dupres, V., Yunus, S., Latgé, J.-P., Heinisch, J.J., Dufrêne, Y.F., 2012. High-Resolution Imaging of Chemical and Biological Sites on Living Cells Using Peak Force Tapping Atomic Force Microscopy. Langmuir 28, $\mathrm{n}^{\mathrm{o}} 49,16738-16744$.

Benech, J.C., Benech, N., Zambrana, A.I., Rauschert, I., Bervejillo, V., Oddone, N., Damián, J.P., 2014. Diabetes increases stiffness of live cardiomyocytes measured by atomic force microscopy nanoindentation. Am J Physiol - Cell Physiol 307, 910-919.

Benech, J.C., Benech, N., Zambrana, A.I., Rauschert, I., Bervejillo, V., Oddone, N., Alberro, A., Damián, J.P., 2015. Intrinsic nanomechanical changes in live diabetic cardiomyocytes. Cardiovascular Regenerative Medicine 2, e893.

Binnig, G., Rohrer, H., Gerber, C., Weibel, E., 1982. Tunneling through a controllable vacuum gap. Appl. Phys. Lett. 40, 178-180.

Binnig, G., Quate, C.F., 1986. Atomic force microscope. Phys. rev. lett. 56, 930-933.

Calzado-Martín, A., Encinar, M., Tamayo, J., Calleja, M., San Paulo, A., 2016. Effect of actin organization on the stiffness of living breast cancer cells revealed by peak-force modulation atomic force microscopy. ACSnano, in press.

Chopinet, L., Formosa, C., Rols, M.P., Duval, R.E., Dague, E., 2013. Imaging living cells surface and quantifying its properties at high resolution using AFM in QITM mode. Micron 48, 26-33.

Cooley, J.W., Tukey, J.W., 1965. An algorithm for the machine calculation of complex Fourier series. Math. Comput. 19, 297-301.

Costa, K.D., 2003. Single-cell elastography: probing for disease with the atomic force microscope. Disease Markers 19, 139-154.

Cross, S.E., Jin, Y.-S., Rao, J., Gimzewski, J.K, 2007. Nanomechanical analysis of cells from cancer patients. Nat. Nanotechnol. 2, 780-783.

Dague, E., Alsteens, D., Latgé, J.-P., Verbelen, C., Raze, D., Baulard, A.R., Dufrêne Y.F., 2007. Chemical force microscopy of single live cells. Nano Lett. 7, n 10, 3026-3030.

Dague, E., Alsteens, D., Latgé, J.-P., Dufrêne Y.F., 2008. High-resolution cell surface dynamics of germination aspergillus fumigatus conidia. Biophysical Journal 94 (2), 656-660.

Dague, E., Genet, G., Lachaize, V., Guilbeau-Frugier, C., Fauconnier, J., Mias, C., Payré, B., Chopinet, L., Alsteens, D., Kasas, S., Severac, C., Thireau, J., Heymes, C., Honton B.,, Lacampagne, A., Pathak, A., Sénard, J.-M., Galés, C., 2014. Atomic force and electron microscopic-based study of sarcolemmal surface of living cardiomyocytes unveils unexpected mitochondrial shift in heart failure. Journal of Molecular and Cellular Cardiology 74, 162-172.

Derjaguin, B.V., Muller, V.M., Toporov, Y.P., 1975. Effect of contact deformations on the adhesion of particles. Journal of Colloid and Interface Science 53(2), 314-326. 
Dokukin M., Sokolov I., 2015. High-resolution high-speed dynamic mechanical spectroscopy of cells and other soft materials with the help of atomic force microscopy. Scientific Reports 5, 12630.

Dufrêne, Y.F., Muller, D.J., 2008. Atomic Force Microscopy as a multifunctional molecular toolbox in nanobiotechnology. Nat. Nanotechnol. 3, 261-269.

Dufrêne, Y.F., Martínez-Martín, D., Medalsy, I., Alsteens, D., Müller, D.J., 2013. Multiparametric imaging of biological systems by force-distance curve-based AFM. Nat. Methods 10, $\mathrm{n}^{\circ}$ 9, 847-854.

Emerson, R.J., Camesano, T.A., 2006. On the importance of precise calibration techniques for an atomic force microscope. Ultramicroscopy 106, 413-422.

Formosa, C., Grare, M., Duval, R.E., Dague, E, 2012a. Nanoscale effects of antibiotics on P. aeruginosa. Nanomed. Nanotechnol. Biol. Med. 8, 12-16.

Formosa, C., Grare, M., Jauvert, E., Coutable, A., Regnouf-de-Vains, J.B., Mourer, M., Duval, R.E., Dague, E., 2012b. Nanoscale analysis of the effects of antibiotics and CX1 on a Pseudomonas aeruginosa multidrugresistant strain. Scientific Reports 2, 575-583.

Formosa, C., Schiavone, M., Boisrame, A., Richard, M.L., Duval, R.E., Dague, E.. 2015. Multiparametric imaging of adhesive nanodomains at the surface of Candida albicans by atomic force microscopy. Nanomedicine 11(1), 57-65.

Genet, G., Guilbeau-Frugier, C., Honton, B., Dague, E., Schneider, M.D., Coatrieux, C., Calise, D., Cardin, C., Nieto, C., Payré, B., Dubroca, C., Marck, P., Heymes, C., Dubrac, A., Arvanitis, D., Despas, F., Altié, M.F., Seguelas, M.H., Delisle, M.B., Davy, A., Sénard, J.M., Pathak, A., Galés, C., 2012. Ephrin-B1 is a novel specific component of the lateral membrane of the cardiomyocyte and is essential for the stability of cardiac tissue architecture cohesion. Circ Res. 110(5), 688-700.

Guilak, F., 2000. The deformation behavior and viscoelastic properties of chondrocytes in articular cartilage. Biorheology 37, 27-44.

Heu, C., Berquand, A., Elie-Caille, C., Nicod, L., 2012. Glyphosate-induced stiffening of HaCaT keratinocytes, a Peak Force Tapping study on living cells. J Struct Biol. 178 (1), 1-7.

Hilal-Dandan, R., Kanter, J.R., Brunton, L.L., 2000. Characterization of G-protein signaling in ventricular myocytes from the adult mouse heart: differences from the rat. J Mol Cell Cardiol. 32, 1211-1221.

Hozic, A., Rico, F., Colom, A., Buzhynskyy, N., Scheuring, S., 2012. Nanomechanical characterization of the stiffness of eye lens cells a pilot study. Investigative Ophthalmology \& Visual Science 53, nº 4, 2151-2156.

Hu, Y., Hu, S., Su, C., Shi, J., Ma, J., 2011. US Patent: US2011/0167524 A1.

Huang, H., Kamm, R.D., Lee, R.T., 2004. Cell mechanics and mechanotransduction: pathways, probes, and physiology. American Journal of Physiology-Cell Physiology 287, C1-C11.

Jacot, J.G., Martin, J.C., Hunt, D.L., 2010. Mechanobiology of cardiomyocyte development. J Biomech 43, $93-$ 98.

Krotil, H.-U., Stifter, T., Waschipky, H., Weishaupt, K., Hild, S., Marti, O., 1999. Pulsed force mode: a new method for the investigation of surface properties. Surf. Interface Anal. 227, 336-340.

Lachaize, V., Formosa, C., Smolyakov, G., Guilbeau-Frugier, C., Galés, C., Dague, E., 2015. Atomic force microscopy: an innovative technology to explore cardiomyocyte cell surface in cardiac physio/pathophysiology. Letters in Applied NanoBioScience 4 (4), 321-334. 
Leu, M., Ehler, E., Perriard, J.C., 2011. Characterisation of postnatal growth of the murine heart . Anat. Embryol. 204, n 3, 217-224.

Lieber, S.C., Aubry, N., Pain, J., Diaz, G., Kim, S.J., Vatner, S.F., 2004. Aging increases stiffness of cardiac myocytes measured by atomic force microscopy nanoindentation. Am J Physiol Heart Circ Physiol 287, H645-651.

Liu, S., Wang, Y., 2010. Application of AFM in microbiology: a review. Scanning 32, 61-73.

Martín-Rodríguez, A.J., González-Orive, A., Hernández-Creus, A., Morales, A., Dorta-Guerra, R., Norte, M., Martín, V.S., Fernández, J.J., 2014. On the influence of the culture conditions in bacterial antifouling bioassays and biofilm properties: Shewanella algae, a case study. BMC Microbiology 14, 102-115.

Muller, D.J., Helenius, J., Alsteens, D., Dufrene, Y.F, 2009. Force probing surfaces of living cells to molecular resolution. Nat. Chem. Biol. 5, 383-390.

Muller, V.M., Derjaguin, B.V., Toporov, Y.P., 1983. On two methods of calculation of the force of sticking of an elastic sphere to a rigid plane. Colloids and Surfaces 7(3), 251-259.

Pillet, F., Chopinet, L., Formosa, C., Dague, E., 2014. Atomic Force Microscopy and pharmacology: from microbiology to cancerology. Biochim Biophys Acta 1840 (3), 1028-1050.

Radmacher, M., Cleveland, J.P., Fritz, M., Hansma, H.G., Hansma, P.K, 1994. Mapping interaction forces with the atomic force microscope. Biophysical Journal 66, 2159-2165.

Slade, A., Pittenger, B., Milani, P., Boudaoud, A., Hamant, O., Kioschis, P., Ponce, L.M., Hafner, M, 2014. Investigating cell mechanics with atomic force microscopy. Microscopy and Analysis 28 (3), S6-S9.

Smolyakov, G., Formosa-Dague, C., Severac, C., Duval, R.E., Dague, E., 2016. High speed indentation measures by FV, QI and QNM introduce a new understanding of bionanomechanical experiments. Micron $85,8-14$

Sneddon, I.N., 1965. The Relation between Load and Penetration in the Axisymmetric Boussinesq Problem for a Punch of Arbitrary Profile. Int. J. Eng. Sci. 3, 47-57. 
(A)

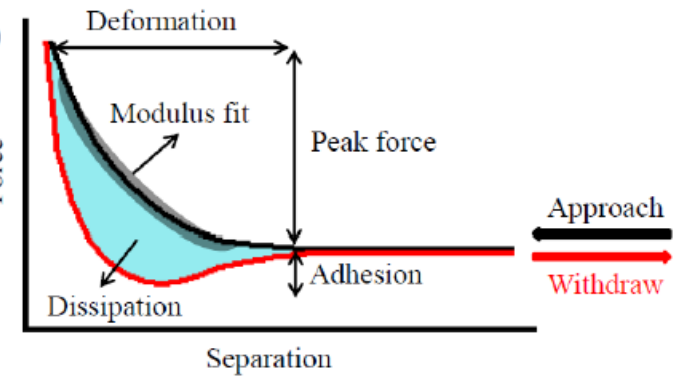

(C)

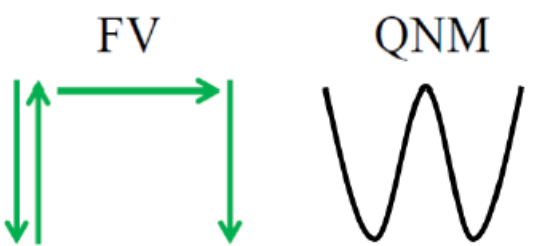

(B)

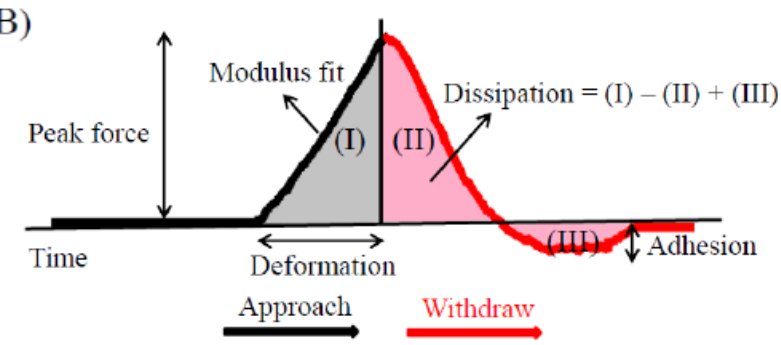

(D)

\begin{tabular}{|c|c|c|}
\hline & FV & QNM \\
Frequency range $(\mathrm{Hz})$ & $0.5 \div 13$ & $250-500$ \\
Acquisition time $(\mathrm{s})$ & $\geq 5$ & $\geq 60$ \\
Image resolution $\left(\mathrm{px}^{2}\right)$ & $8^{2} \div 64^{2}$ & $128^{2} \div 512^{2}$ \\
Number of curves by pixel & 1 & $1-20$ \\
\hline
\end{tabular}

Fig. 1. Force curves used to calculate the mechanical and adhesive properties: in representations force vs. separation (A) and force $v s$. time (B); schema of the tip pathway in the FV and PeakForce QNM modes (C); comparative table for the two modes (D). 


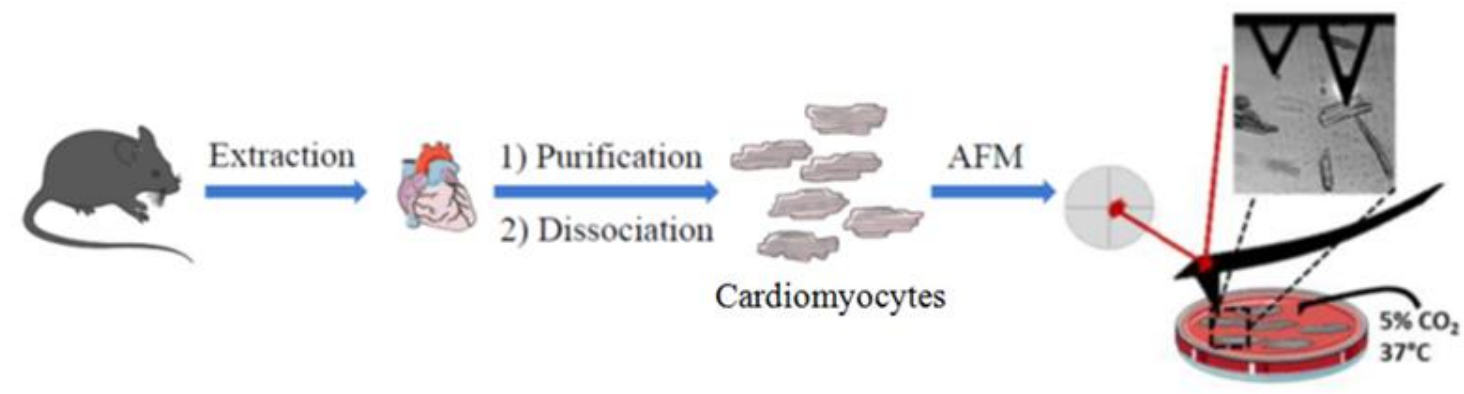

Fig. 2. Schematic representation of the preparation of living CMs for AFM measurements. 

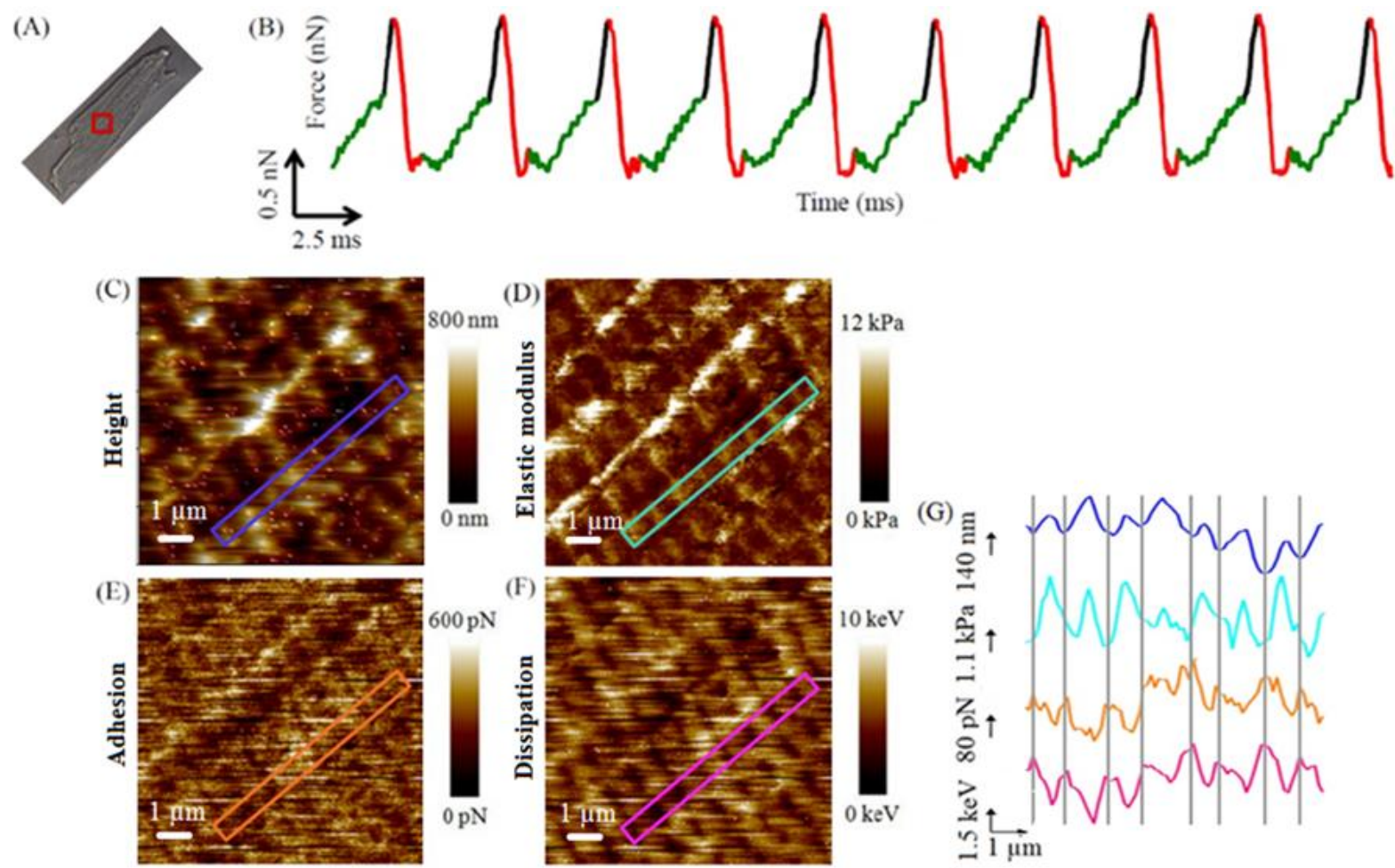

Fig. 3. PeakForce QNM analysis of a living cardiomyocyte surface using the hydrophobic tip. CM optical image indicating the AFM scanned area (red) (A); force curves in the force vs. time representation with the approach contact part in black, the withdraw contact part in red and the noncontact part in green (B); representative maps ( $128^{2}$ pixels resolution) of 3D height (C), Sneddon elastic modulus (D), adhesion (E) and dissipation (F) obtained from the scanned area shown on the optical image in (A); correlation between the height (blue), Sneddon elastic modulus (cyan), adhesion (orange) and dissipation (rose) traces along the boxes shown on the corresponding maps (G). 

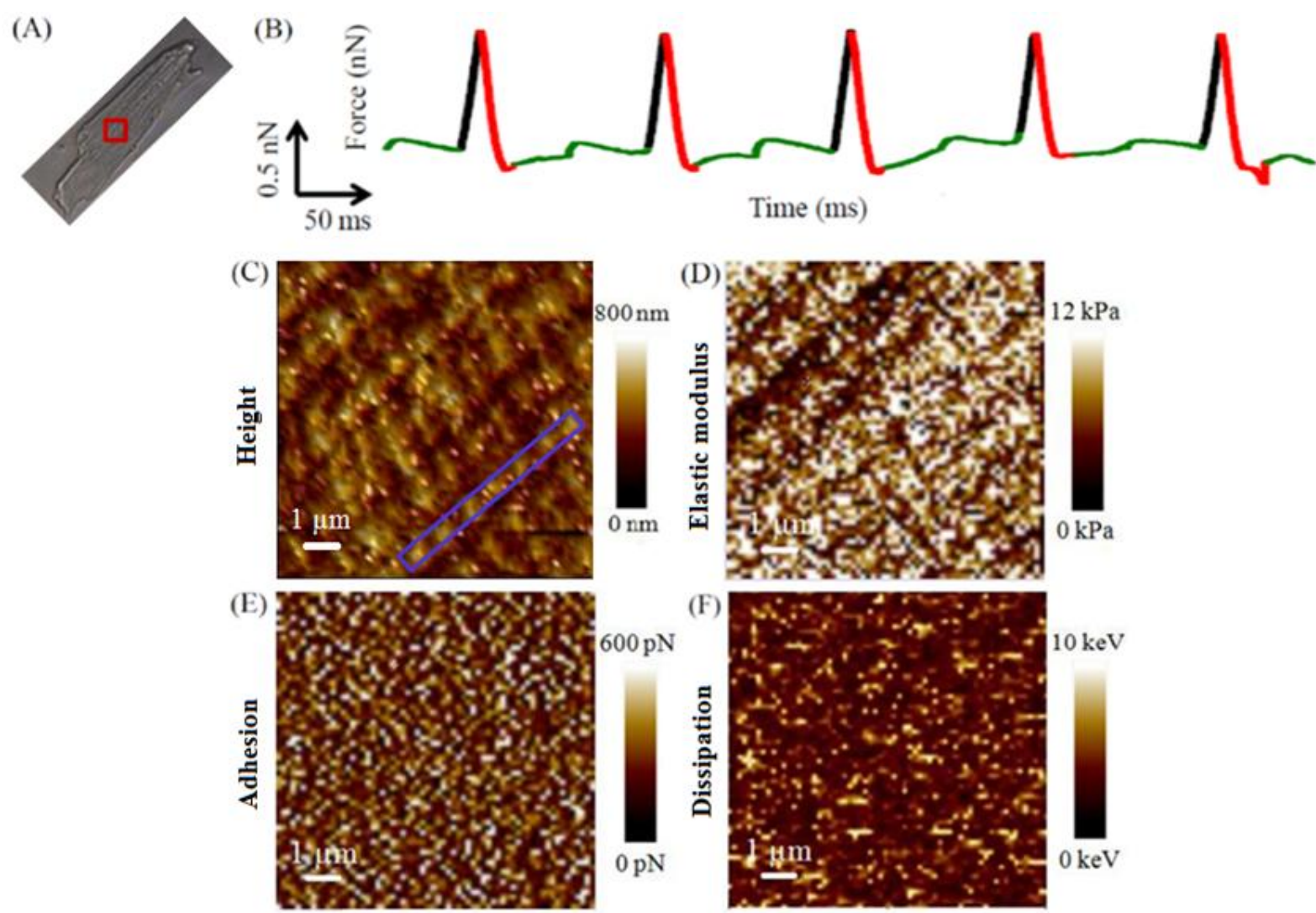

(G)

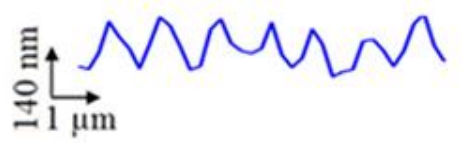

Fig. 4. FV analysis of a living cardiomyocyte surface using the hydrophobic tip. CM optical image indicating the AFM scanned area (red) (A); force curves in the force vs. time representation with the approach contact part in black, the withdraw contact part in red and the noncontact part in green (B); representative maps ( $64^{2}$ pixels resolution) of 3D height (C), Sneddon elastic modulus (D), adhesion (E) and dissipation (F) obtained from the scanned area shown on the optical image (A) and trace along the box shown on the height map $(\mathrm{G})$. 


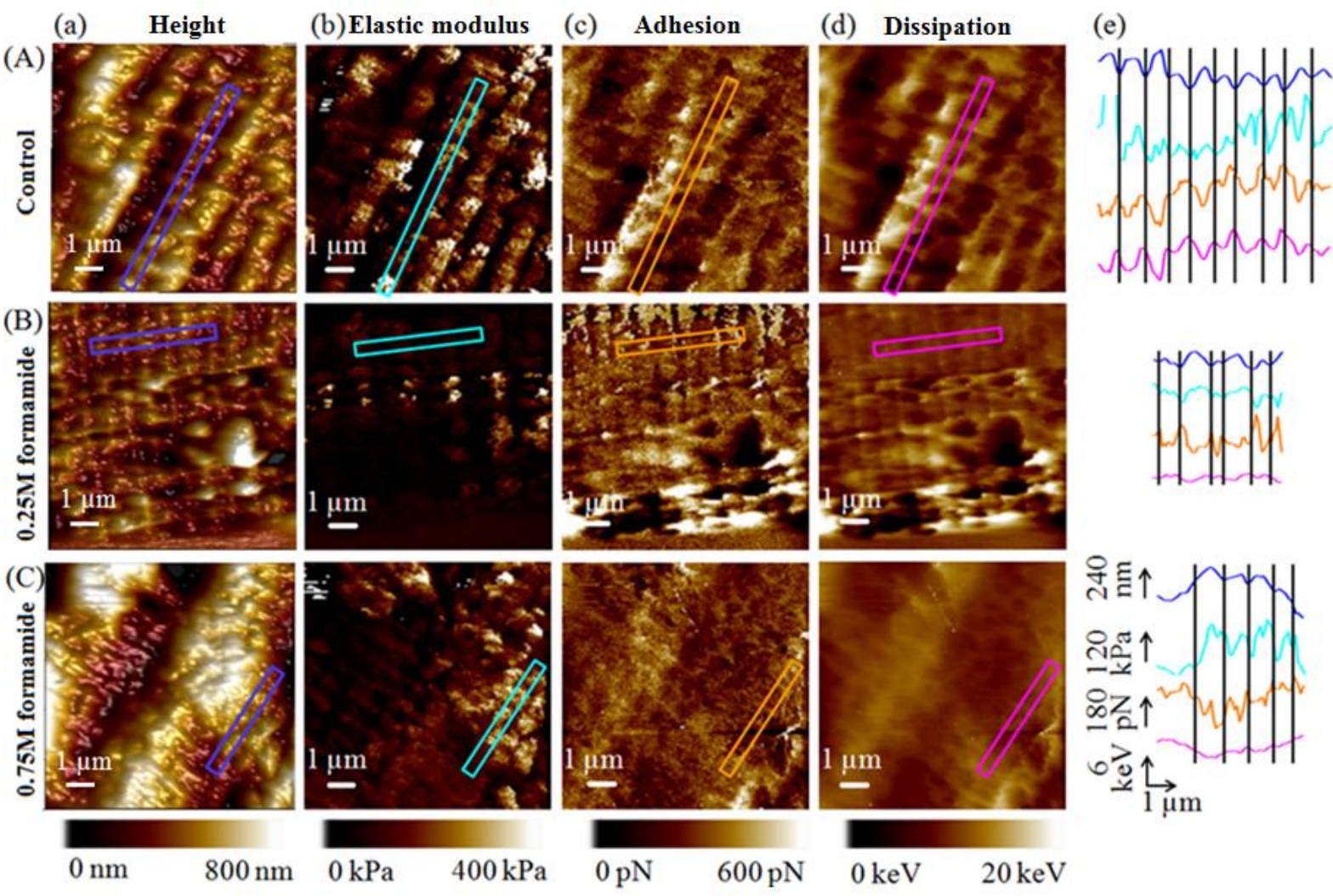

Fig. 5. PeakForce QNM on fixed cardiomyocyte surface using the hydrophobic tip. Control CM (A), CM pretreated with $0.25 \mathrm{M}$ (B) and $0.75 \mathrm{M}$ (C) formamide. In each case, maps $\left(128^{2}\right.$ pixels resolution) of the 3D height (a), DMT elastic modulus (b), adhesion (c) and dissipation (d) are shown, as well as correlation between the height (blue), DMT elastic modulus (cyan), adhesion (orange) and dissipation (rose) along the boxes shown on the corresponding maps (e) 


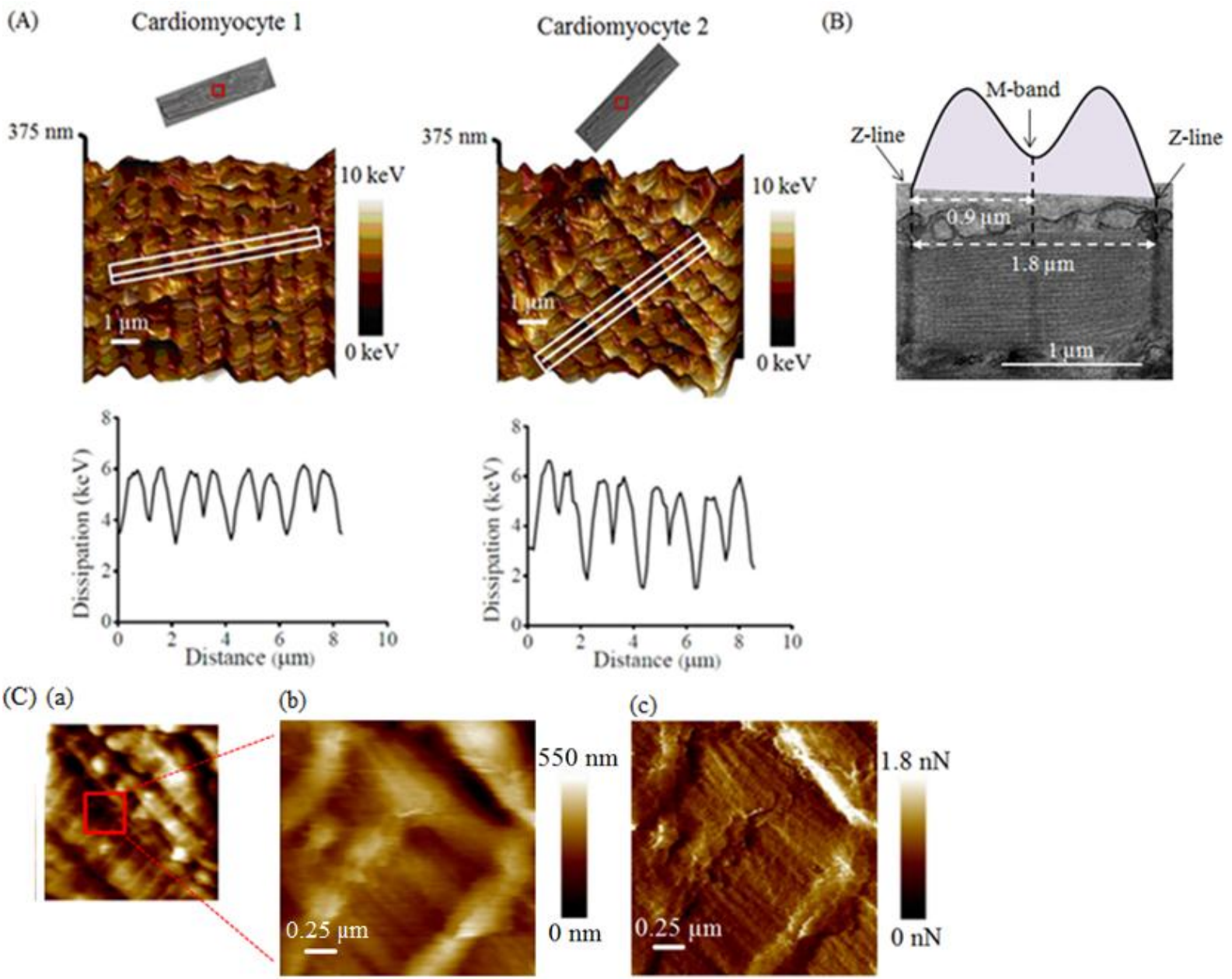

Fig. 6. PeakForce QNM analysis of cardiomyocyte ultrastructures. Dissipation maps (colour) superimposed with 3D height maps (relief) (done as shown by red squares on the CMs optical images) with corresponding traces (done as shown on the maps) obtained for two different fixed CMs and pretreated with $0.25 \mathrm{M}$ formamide (A); schematic superimposition of a dissipitation map illustrating the camel structure and an electron microscopy micrograph illustrating the CM sarcomeric structure (B); representative height map $\left(10^{2} \mu \mathrm{m}^{2}\right)(\mathrm{Ca})$ and its $2.2^{2} \mu \mathrm{m}^{2}$ zoom $(\mathrm{Cb})$ and PeakForce error $(\mathrm{Cc})$. All maps were obtained by PeakForce QNM using hydrophobic tip with a $128^{2}$ pixels resolution. 

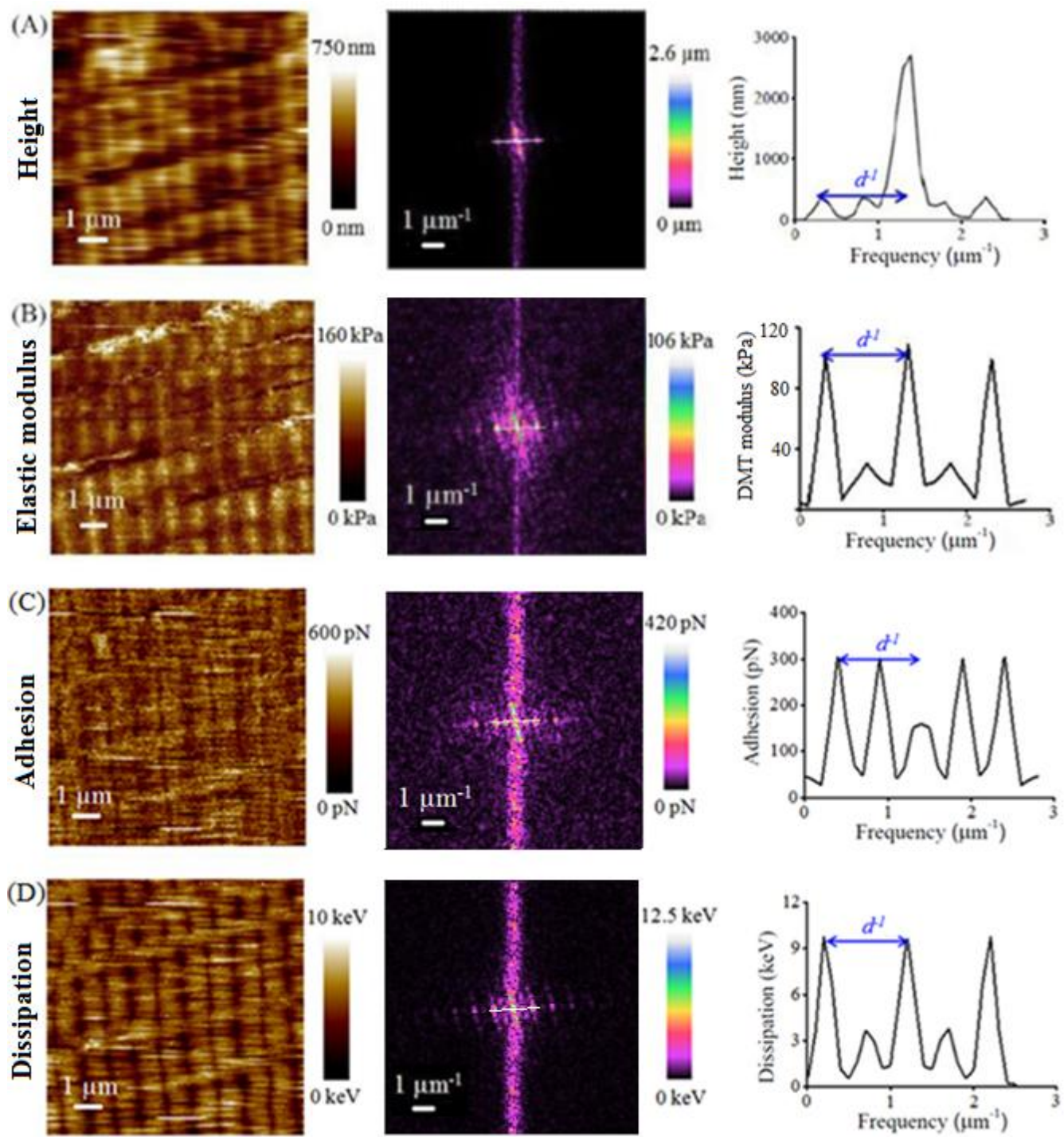

Fig. 7. PeakForce QNM cartography $\left(128^{2}\right.$ pixels resolution) using the hydrophobic tip. For each mechanical map obtained on a fixed CM pretreated with $0.25 \mathrm{M}$ formamide, the corresponding twodimensional Fast Fourier Transform (2D-FFT) images and derived traces are shown. Height (A), DMT elastic modulus (B), adhesion (C) and dissipation (D) maps. The calculated distance between peaks is $d=1.00 \mu \mathrm{m}$. 